\title{
RELATIVE DEFECTS CORRESPONDING TO THE COMMON ROOTS OF TWO MEROMORPHIC FUNCTIONS
}

\author{
By ANAND PRAKASH Singh
}

I. Introduction. The concept of absolute defect of $a$ with respect to the derivative $f^{\prime}$ was introduced by $H$. Milloux [2]. This definition was later extended by Xiong Qing-Lai [4]. He introduced the term

$$
\delta_{r}^{(k)}(a, f)=1-\limsup _{r \rightarrow \infty} \frac{N\left(r, 1 / f^{(k)}-a\right)}{T(r, f)}
$$

and called it as the relative defect of the value $a$ with respect to the derivative $f^{(k)}$. And the usual defect with respect to $f^{(k)}$ viz

$$
\delta_{a}^{(k)}(a, f)=1-\limsup _{r \rightarrow \infty} \frac{N\left(r, 1 / f^{(k)}-a\right)}{T\left(r, f^{(k)}\right)}
$$

was called the absolute defect of the value $a$ with respect to $f^{(k)}$. In this paper he found various relations between these two defects. Later A.P. Singh [3] defined the relative defects corresponding to distinct zeros and distinct poles and found various relations between these. In the present paper we shall consider two different meromorphic functions having common roots and find some relations involving the relative defects. We assume that the reader has some familiarity with the Nevanlinna theory of meromorphic functions. See for example [1].

II. Notations, Terminology. Let $f_{1}(z), f_{2}(z)$ be two non-constant meromorphic functions and let $a$ be any complex number. Let $n_{0}(r, a)$ denote the number of common roots in the disk $|z| \leqq r$ of the two equations $f_{1}(z)=a$ and $f_{2}(z)=a$, and let $\bar{n}_{0}(r, a)$ denote the number of common roots in the disk $|z| \leqq r$ of the two equations $f_{1}(z)=a$ and $f_{2}(z)=a$, where the multiplicity is disregarded (i. e. each root being counted only once). Set

$$
\begin{array}{r}
\bar{N}_{0}(r, a)=\int_{0}^{r} \frac{\bar{n}_{0}(t, a)-\bar{n}_{0}(0, a)}{t} d t+\bar{n}_{0}(0, a) \log r, \\
\bar{N}_{1,2}(r, a)=\bar{N}\left(r, \frac{1}{f_{1}-a}\right)+\bar{N}\left(r, \frac{1}{f_{2}-a}\right)-2 \bar{N}_{0}(r, a) .
\end{array}
$$

Let $\bar{n}_{0}^{(k)}(r, a), \bar{N}_{1,2}^{(k)}(r, a)$ etc. denote the corresponding quantities with respect to $f_{1}^{(k)}$ and $f_{2}^{(k)}$. Set

Received November 8, 1982 


$$
\begin{aligned}
& \Theta_{1,2}(a)=1-\limsup _{r \rightarrow \infty} \frac{\bar{N}_{1,2}(r, a)}{T\left(r, f_{1}\right)+T\left(r, f_{2}\right)}, \\
& \Theta_{1,2}^{(k)}(a)=1-\limsup _{r \rightarrow \infty} \frac{\bar{N}_{1,2}^{(k)}(r, a)}{T\left(r, f_{1}\right)+T\left(r, f_{2}\right)}, \\
& \delta_{1,2}(a)=1-\limsup _{r \rightarrow \infty} \frac{N_{1,2}(r, a)}{T\left(r, f_{1}\right)+T\left(r, f_{2}\right)},
\end{aligned}
$$

$\Theta_{0}(a), \Theta_{0}^{(k)}(a)$ being similarly defined. The term $S(r, f)$ will denote any quantity satisfying $S(r, f)=o(T(r, f))$ as $r \rightarrow \infty$ except possibly for a set of $r$ of finite linear measure. For other usual notations see for e.g. [1].

\section{Results.}

THEOREM 1. Let $f_{1}(z), f_{2}(z)$ be two meromorphic functions such that $N\left(r, \frac{1}{f_{1}}\right)$ $=S\left(r, f_{1}\right)$ and $N\left(r, \frac{1}{f_{2}}\right)=S\left(r, f_{2}\right)$. Then for any $a \neq 0, \infty$

$$
\Theta_{1,2}^{(k)}(a)+2 \Theta_{0}^{(k)}(a) \leqq 5-\left(\Theta_{1,2}(\infty)+2 \Theta_{0}(\infty)\right) .
$$

For the proof we shall need the following lemma of Milloux $[1,55]$.

LEMMA. Let $k$ be a positive integer and let $\emptyset(z)=\sum_{i=0}^{k} a_{i}(z) f^{(i)}(z)$ where $a_{i}(z)$ are meromorphic functions such that $T\left(r, a_{i}(z)\right)=S(r, f)$, then $m\left(r, \frac{\emptyset(z)}{f(z)}\right)=S(r, f)$ and $T(r, 0) \leqq(k+1) T(r, f)+S(r, f)$.

Proof of theorem. For any meromorphic function $f$ and a complex number $a \neq 0, \infty$ we first consider the following identity

$$
\frac{a}{f}=\frac{f^{(k)}}{f}-\frac{f^{(k)}-a}{f^{(k+1)}} \cdot \frac{f^{(k+1)}}{f}
$$

By the lemma one easily gets

$$
\begin{aligned}
m\left(r, \frac{a}{f}\right) & \leqq m\left(r, \frac{f^{(k)}-a}{f^{(k+1)}}\right)+S(r, f) \\
& =T\left(r, \frac{f^{(k)}-a}{f^{(k+1)}}\right)-N\left(r, \frac{f^{(k)}-a}{f^{(k+1)}}\right)+S(r, f) .
\end{aligned}
$$

By Nevanlinna's first theorem and the lemma we get

$$
m\left(r, \frac{a}{f}\right) \leqq N\left(r, \frac{f^{(k+1)}}{f^{(k)}-a}\right)-N\left(r, \frac{f^{(k)}-a}{f^{(k+1)}}\right)+S(r, f) .
$$

And so by $[1,34]$ we get 


$$
\begin{aligned}
m\left(r, \frac{a}{f}\right) \leqq N\left(r, f^{(k+1)}\right) & +N\left(r, \frac{1}{f^{(k)}-a}\right)-N\left(r, f^{(k)}-a\right) \\
& -N\left(r, \frac{1}{f^{(k+1)}}\right)+S(r, f) .
\end{aligned}
$$

And so

$$
\begin{aligned}
T(r, f)= & m\left(r, \frac{a}{f}\right)+N\left(r, \frac{a}{f}\right)+S(r, f) \\
\leqq & N\left(r, f^{(k+1)}\right)+N\left(r, \frac{1}{f^{(k)}-a}\right)-N\left(r, f^{(k)}-a\right) \\
& -N\left(r, \frac{1}{f^{(k+1)}}\right)+N\left(r, \frac{a}{f}\right)+S(r, f) .
\end{aligned}
$$

Also it can easily be verified that $N\left(r, f^{(k)}-a\right)=N\left(r, f^{(k)}\right), N\left(r, \frac{1}{f^{(k)}-a}\right)-$ $N\left(r, \frac{1}{f^{(k+1)}}\right) \leqq \bar{N}\left(r, \frac{1}{f^{(k)}-a}\right), N\left(r, f^{(k+1)}\right)-N\left(r, f^{(k)}\right)=\bar{N}\left(r, f^{(k)}\right)$ and $\bar{N}\left(r, f^{(k)}\right)$ $=N(r, f)$. Applying these results to the above inequality we get

$$
T(r, f) \leqq \bar{N}\left(r, \frac{1}{f^{(k)}-a}\right)+\bar{N}(r, f)+N\left(r, \frac{1}{f}\right)+S(r, f) .
$$

Applying this inequality for $f_{1}$ and $f_{2}$ and using the hypothesis we get

$$
\begin{aligned}
T\left(r, f_{1}\right)+T\left(r, f_{2}\right) \leqq & \bar{N}\left(r, \frac{1}{f_{1}^{(k)}-a}\right)+\bar{N}\left(r, \frac{1}{f_{2}^{(k)}-a}\right)+\bar{N}\left(r, f_{1}\right)+\bar{N}\left(r, f_{2}\right) \\
& +S\left(r, f_{1}\right)+S\left(r, f_{2}\right) \\
=\bar{N}_{1,2}^{(k)}(r, a) & +2 \bar{N}_{0}^{(k)}(r, a)+\bar{N}_{1,2}(r, \infty) \\
& +2 \bar{N}_{0}(r, \infty)+S\left(r, f_{1}\right)+S\left(r, f_{2}\right) .
\end{aligned}
$$

The result now follows by dividing with $T\left(r, f_{1}\right)+T\left(r, f_{2}\right)$ and taking limit superior as $r \rightarrow \infty$.

Remark. Since the poles of $f$ and the poles of $f^{(k)}$ occur at the same point, it follows that $\bar{N}\left(r, f^{(k)}\right)=\bar{N}(r, f)$ and so $\Theta_{1,2}^{(k)}(\infty)$ etc. reduce to $\Theta_{1,2}(\infty)$ respectively. However in this case we can show

THEOREM 2. Let $f_{1}(z)$ and $f_{2}(z)$ be any two meromorphic functions with $\bar{N}\left(r, \frac{1}{f_{1}}\right)=S\left(r, f_{1}\right)$ and $\bar{N}\left(r, \frac{1}{f_{2}}\right)=S\left(r, f_{2}\right)$. Then for any $a \neq 0, \infty$

$$
\Theta_{1,2}(\infty)+2 \Theta_{0}(\infty) \leqq 5-\left(\Theta_{1,2}(a)+2 \Theta_{0}(a)\right) .
$$

The proof of the above theorem follows as in the previous theorem by considering the inequality (see for e.g. inequality $2.9[1,43]$ ) 


$$
T(r, f) \leqq \bar{N}(r, f)+\bar{N}\left(r, \frac{1}{f}\right)+\bar{N}\left(r, \frac{1}{f-a}\right)+S(r, f) .
$$

From the above inequality we also have for all non-negative integers $k$,

$$
\begin{aligned}
T(r, f) & \leqq k \bar{N}(r, f)+N(r, f)+\bar{N}\left(r, \frac{1}{f}\right)+\bar{N}\left(r, \frac{1}{f-a}\right)+S(r, f) \\
& =N\left(r, f^{(k)}\right)+\bar{N}\left(r, \frac{1}{f}\right)+\bar{N}\left(r, \frac{1}{f-a}\right)+S(r, f),
\end{aligned}
$$

which, when applied to the two functions $f_{1}$ and $f_{2}$, will yield

THEOREM 3. Let $f_{1}(z)$ and $f_{2}(z)$ be any two meromorphic functions with $\bar{N}\left(r, \frac{1}{f_{1}}\right)=S\left(r, f_{1}\right)$ and $\bar{N}\left(r, \frac{1}{f_{2}}\right)=S\left(r, f_{2}\right)$. Then for any $a \neq 0, \infty$

$$
\delta_{1,2}^{(k)}(\infty)+2 \delta_{0}^{(k)}(\infty) \leqq 5-\left(\Theta_{1,2}(a)+2 \Theta_{0}(a)\right) .
$$

THEOREM 4. Let $f_{1}$ and $f_{2}$ be two meromorphic functions such that $N\left(r, \frac{1}{f_{1}}\right)$ $=S\left(r, f_{1}\right)$ and $N\left(r, \frac{1}{f_{2}}\right)=S\left(r, f_{2}\right)$. Then for any finite, non zero, distinct $a, b$

$$
\Theta_{1,2}^{(k)}(a)+\Theta_{1,2}^{(k)}(b) \leqq 5-2\left(\Theta_{0}^{(k)}(a)+\Theta_{0}^{(k)}(b)\right) .
$$

Proof. For any meromorphic function $f$ we have

$$
\begin{aligned}
m\left(r, \frac{1}{f}\right) & \leqq m\left(r, \frac{f^{(k)}}{f}\right)+m\left(r, \frac{1}{f^{(k)}}\right) \\
& =m\left(r, \frac{1}{f^{(k)}}\right)+S(r, f) \quad \text { by the lemma } \\
& =T\left(r, \frac{1}{f^{(k)}}\right)-N\left(r, \frac{1}{f^{(k)}}\right)+S(r, f) .
\end{aligned}
$$

And hence by Nevanlinna's first and second theorem we get

$$
\begin{aligned}
m\left(r, \frac{1}{f}\right) \leqq & \bar{N}\left(r, \frac{1}{f^{(k)}}\right)+\bar{N}\left(r, \frac{1}{f^{(k)}-a}\right)+\bar{N}\left(r, \frac{1}{f^{(k)}-b}\right) \\
& -N\left(r, \frac{1}{f^{(k)}}\right)+S(r, f) \\
\leqq & \bar{N}\left(r, \frac{1}{f^{(k)}-a}\right)+\bar{N}\left(r, \frac{1}{f^{(k)}-b}\right)+S(r, f) .
\end{aligned}
$$

And so it follows that

$$
T(r, f) \leqq \bar{N}\left(r, \frac{1}{f^{(k)}-a}\right)+\bar{N}\left(r, \frac{1}{f^{(k)}-b}\right)+N\left(r, \frac{1}{f}\right)+S(r, f) .
$$


Applying (1) for $f_{1}$ and $f_{2}$ and since $N\left(r, \frac{1}{f_{1}}\right)=S\left(r, f_{1}\right)$ and $N\left(r, \frac{1}{f_{2}}\right)=S\left(r, f_{2}\right)$ we get

$$
\begin{aligned}
T\left(r, f_{1}\right)+T\left(r, f_{2}\right) \leqq & \bar{N}\left(r, \frac{1}{f_{1}^{(k)}-a}\right)+\bar{N}\left(r, \frac{1}{f_{2}^{(k)}-a}\right)+\bar{N}\left(r, \frac{1}{f_{1}^{(k)}-b}\right) \\
+ & \bar{N}\left(r, \frac{1}{f_{2}^{(k)}-b}\right)+S\left(r, f_{1}\right)+S\left(r, f_{2}\right) \\
= & \bar{N}_{1,2}^{(k)}(r, a)+\bar{N}_{1,2}^{(k)}(r, b)+2 \bar{N}_{0}^{(k)}(r, a) \\
& \quad+2 \bar{N}_{0}^{(k)}(r, b)+S\left(r, f_{1}\right)+S\left(r, f_{2}\right) .
\end{aligned}
$$

The result now follows by dividing with $T\left(r, f_{1}\right)+T\left(r, f_{2}\right)$ and taking limit superior as $r \rightarrow \infty$.

We now prove

THEOREM 5. Let $f_{1}(z)$ and $f_{2}(z)$ be two meromorphic functions which have 0 and $\infty$ as exceptional value of defect 1 . Let $a_{\imath}$ be finite, distınct, non-zero complex numbers, then $\sum_{\imath} \Theta_{1,2}^{(k)}\left(a_{\imath}\right) \leqq 2$. $p \geqq 1$,

Proof. By an inequality from theorem 7 of [3], we have for all integers

$$
\begin{aligned}
p T(r, f) \leqq & p N\left(r, \frac{1}{f}\right)+\bar{N}(r, f)+\bar{N}\left(r, \frac{1}{f^{(k)}}\right)+\sum_{i=1}^{p} \bar{N}\left(r, \frac{1}{f^{(k)}-a_{i}}\right) \\
& -p N\left(r, \frac{1}{f^{(k)}}\right)+S(r, f) .
\end{aligned}
$$

Now clearly $\bar{N}\left(r, \frac{1}{f^{(k)}}\right)-p N\left(r, \frac{1}{f^{(k)}}\right) \leqq 0$ and if $\delta(0, f)=\delta(\infty, f)=1$, then the above inequality reduces to

$$
p T(r, f) \leqq \sum_{i=1}^{p} \bar{N}\left(r, \frac{1}{f^{(k)}-a_{i}}\right)+S(r, f) .
$$

Using this to our two functions $f_{1}$ and $f_{2}$ we get

$$
\begin{aligned}
p\left(T\left(r, f_{1}\right)+T\left(r, f_{2}\right)\right) \leqq & \sum_{\imath=1}^{p}\left(\bar{N}\left(r, \frac{1}{f_{1}^{(k)}-a_{\imath}}\right)+\bar{N}\left(r, \frac{1}{f_{2}^{(k)}-a_{\imath}}\right)\right) \\
& +S\left(r, f_{1}\right)+S\left(r, f_{2}\right) \\
= & \sum_{\imath=1}^{p} \bar{N}_{1,2}^{(k)}\left(r, a_{\imath}\right)+2 \sum_{\imath=1}^{p} \bar{N}_{0}^{(k)}\left(r, a_{\imath}\right) \\
& +S\left(r, f_{1}\right)+S\left(r, f_{2}\right)
\end{aligned}
$$

Now a common root of $f_{1}^{(k)}=a_{\imath}$ and $f_{2}^{(k)}=a_{\imath}$ is a pole of $\frac{1}{f_{1}^{(k)}-f_{2}^{(k)}}$ and so we 
have

$$
\begin{aligned}
\sum_{i=1}^{p} \bar{N}_{0}^{(k)}\left(r, a_{\imath}\right) & \leqq N\left(r, \frac{1}{f_{1}^{(k)}-f_{2}^{(k)}}\right) \\
& \leqq T\left(r, \frac{1}{f_{1}^{(k)}-\frac{1}{f_{2}^{(k)}}}\right)
\end{aligned}
$$

and thus

$$
\sum_{i=1}^{p} \bar{N}_{0}^{(k)}\left(r, a_{\imath}\right) \leqq T\left(r, f_{1}^{(k)}\right)+T\left(r, f_{2}^{(k)}\right)+O(1) .
$$

Now $\delta\left(0, f_{\imath}\right)=\delta\left(\infty, f_{\imath}\right)=1$ so that $T\left(r, f_{\imath}^{(k)}\right) \sim T\left(r, f_{\imath}\right)$ for $i=1,2$. So from (2) and (3) we get

$$
(p-2)\left(T\left(r, f_{1}\right)+T\left(r, f_{2}\right)\right) \leqq \sum_{\imath=1}^{p} \bar{N}_{1,2}^{(k)}\left(r, a_{\imath}\right)+S\left(r, f_{1}\right)+S\left(r, f_{2}\right) .
$$

Once again, dividing by $T\left(r, f_{1}\right)+T\left(r, f_{2}\right)$ and taking limit superior we obtain $\sum_{\imath=1}^{p} \Theta_{1,2}^{(k)}\left(a_{\imath}\right) \leqq 2$. The result now follows by allowing $p$ to tend to $\infty$.

Finally, we prove a theorem regarding the distinct and the common poles of $f_{1}$ and $f_{2}$.

THEOREM 6. Let $f_{1}$ and $f_{2}$ be two meromorphic functions of finite order and let $T\left(r, f_{\imath}^{\prime}\right) \sim a T\left(r, f_{\imath}\right)$ where $a \geqq 1$ and $i=1,2$. Then

$$
\Theta_{1,2}(\infty)+2 \Theta_{0}(\infty) \leqq 4-a .
$$

Proof. Since $f_{\imath}$ are of finite order it follows that

$$
m\left(r, f_{\imath}^{\prime}\right) \leqq m\left(r, f_{\imath}\right)+O(\log r)
$$

And so

$$
\begin{aligned}
T\left(r, f_{\imath}^{\prime}\right) & =N\left(r, f_{\imath}^{\prime}\right)+m\left(r, f_{\imath}^{\prime}\right) \\
& \leqq N\left(r, f_{\imath}\right)+\bar{N}\left(r, f_{\imath}\right)+m\left(r, f_{\imath}\right)+O(\log r) \\
& =T\left(r, f_{\imath}\right)+\bar{N}\left(r, f_{\imath}\right)+O(\log r) .
\end{aligned}
$$

But by hypothesis $T\left(r, f_{\imath}^{\prime}\right) \sim a T\left(r, f_{\imath}\right)$ and so

$$
(a-1) T\left(r, f_{\imath}\right) \leqq \bar{N}\left(r, f_{\imath}\right)+O(\log r) .
$$

Thus

$$
\begin{aligned}
(a-1)\left(T\left(r, f_{1}\right)+T\left(r, f_{2}\right)\right) & \leqq \bar{N}\left(r, f_{1}\right)+\bar{N}\left(r, f_{2}\right)+O(\log r) \\
& =\bar{N}_{1,2}(r, \infty)+2 \bar{N}_{0}(r, \infty)+O(\log r)
\end{aligned}
$$

and the result follows as earlier. 


\section{REFERENCES}

[1] Hayman, W. K., Meromorphic functions, Oxford University Press, London (1964).

[2] Milloux, H., Lee dérivées des fonctions méromorphes et la théorie des défauts, Ecole Normale Superieure 63 No. 3 (1946), 289-316.

[3] SingH, A.P., Relative defects of meromorphic functions, Journal of the Indian Math. Soc. 44 (1979), 191-202.

[4] XIONG, Qing-LAI, A fundamental inequality in the theory of meromorphic functions, Chinese Mathematics 9 No. 1 (1967), 146-167.

Department of Mathematics

SHIVAJI UnIVERSITY

KOLHAPUR-416004 MAHARASHTRA

INDIA 